\title{
Flavor Correlations and the QCD Phase Structure
}

\author{
Chihiro Sasaki ${ }^{1, a}$ \\ ${ }^{1}$ Institute of Theoretical Physics, University of Wroclaw, PL-50204 Wroclaw, Poland
}

\begin{abstract}
We show a set of fluctuations and correlations associated with physical observables as a probe of a phase transition or its remnant. A possible phase structure of hot and dense QCD is also discussed based on the effective theory approach.
\end{abstract}

\section{Introduction}

Modifications in magnitude of fluctuations for different observables are an excellent probe of a phase transition or its remnant [1]. In particular, fluctuations related to conserved charges carried by light and strange quarks play an important role in the vicinity of the QCD chiral and deconfinement crossover. In this contribution, we show selected susceptibilities to characterize the QCD phase transition and give a brief description of hot/dense QCD matter and the phase structure expected from available effective-theories.

\section{Heavy-light flavor correlations}

Heavy flavors are produced at the initial stage of the high-energy heavy-ion collisions, so that they are expected to carry the dynamical history of a created matter, the Quark-Gluon Plasma (QGP). Recent experimental observations have revealed that charm quarks are thermalized [2-8], contrary to earlier anticipation. Charge fluctuations calculated in lattice QCD also indicate that the charmed mesons are deconfined together with light-flavor mesons [9]. Given those observations, comprehensive exploration for the chiral aspects of the heavy-light hadrons increases its importance.

In constructing effective Lagrangians for the heavy-light mesons, besides spontaneous chiral symmetry breaking, heavy quark symmetry is a vital ingredient [10]. The pseudo-scalar $D$ and vector $D^{*}$ states fill in the same multiplet $H$, forming the lowest spin partners. Their low-energy dynamics is dominated by interactions with Nambu-Goldstone (NG) bosons, pions [11-14]. Introducing the multiplet including $D$ and $D^{*}$ inevitably accompanies another multiplet $G$ which contains a scalar $D_{0}^{*}$ and axial-vector $D_{1}$ states. Those parity partners, $H$ and $G$, become degenerate when the chiral symmetry is restored $[15,16]$.

Aside from the chiral $S U(4)$ approach where the charm sector suffers from a huge explicit breaking of the extended flavor symmetry, a self-consistent study for the thermal charmed-mesons with implementing heavy quark symmetry has received little attention. In Ref. [17], a chiral effective theory for the light and heavy-light mesons has been formulated in the presence of a medium. Below,

\footnotetext{
a e-mail: chihiro.sasaki@ift.uni.wroc.pl
} 

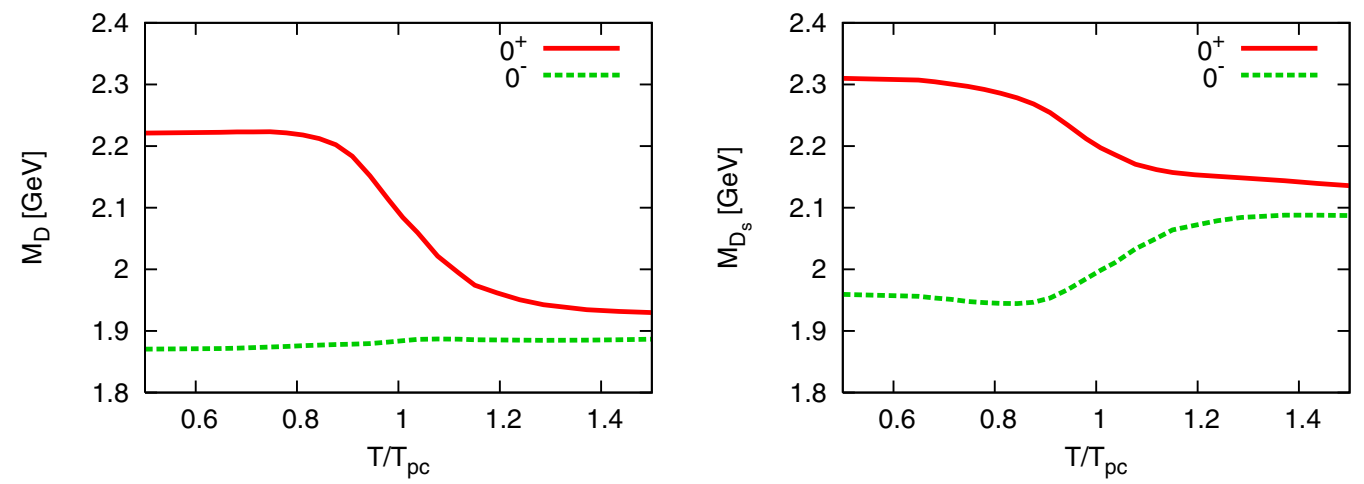

Figure 1. In-medium masses of the non-strange (left) and strange (right) charmed-mesons with positive and negative parity [17].

we will briefly discuss in-medium masses of the heavy-light mesons and the correlations among light and heavy flavors near the chiral crossover.

When the charmed-meson mean fields are introduced to a chiral effective theory in the standard fashion, they act as an extra source which breaks the chiral symmetry explicitly. Consequently, an unrealistically strong mixing between the light-flavor and the charmed meson sector is induced. This defect can be avoided if effective interactions depending on temperature are introduced. Those intrinsic modifications can be extracted from the chiral condensates calculated in lattice QCD. The obtained coupling of the strange charmed meson to the sigma meson, $g_{\pi}^{s}(T)$, becomes quenched as temperature is increased toward the chiral pseudo-critical point $T_{\mathrm{pc}}=154 \mathrm{MeV}$.

Effective charmed-meson masses in hot matter are shown in Fig. 1. The parity partners approach each other as temperature is increased both in the non-strange and strange sector, in consistent with the chiral symmetry restoration. The two pseudo-scalar states have the same trend that their masses are increasing with temperature, although the non-strange meson mass exhibits a rather weak modification. On the other hand, the two scalar states drop significantly; the non-strange meson mass by $\sim 200 \mathrm{MeV}$ and the strange meson mass by $\sim 100 \mathrm{MeV}$. The mass splittings between the nonstrange and strange states are around $200 \mathrm{MeV}$ above $T_{\mathrm{pc}}$ due to the fact that the chiral symmetry in the strange sector is not restored yet. Nevertheless, the chiral mass splittings between the scalar and pseudo-scalar states are almost of the same size,

$$
\delta M_{D}\left(T_{\mathrm{pc}}\right) \sim \delta M_{D_{s}}\left(T_{\mathrm{pc}}\right) \sim 200 \mathrm{MeV},
$$

i.e. the chiral mass differences in the heavy-light sector are blind to the light flavors. This is a striking difference from the chiral properties of the light mesons, and is attributed to the heavy quark symmetry possessed by the leading-order Lagrangian in $1 / m_{Q}$ expansion. In contrast, the chiral SU(4) model, where the charmed mesons are treated on the equal footing to the non-strange and strange mesons, yields a qualitatively different result from Eq. (1); $\delta M_{D}$ is much smaller than $\delta M_{D_{s}}$, similar to the light meson masses [18].

The quenched $g_{\pi}^{s}(T)$ leads also to a strong suppression of the scalar $D_{s}$ decay toward $T_{\mathrm{pc}}$, on top of the suppression due to the small isospin violation. Thus, such an anomalous suppression, if it would be observed, is a signature of chiral symmetry restoration. The same should be carried over to the $B$ and $B_{s}$ mesons with which the heavy quark symmetry is more reliable. 

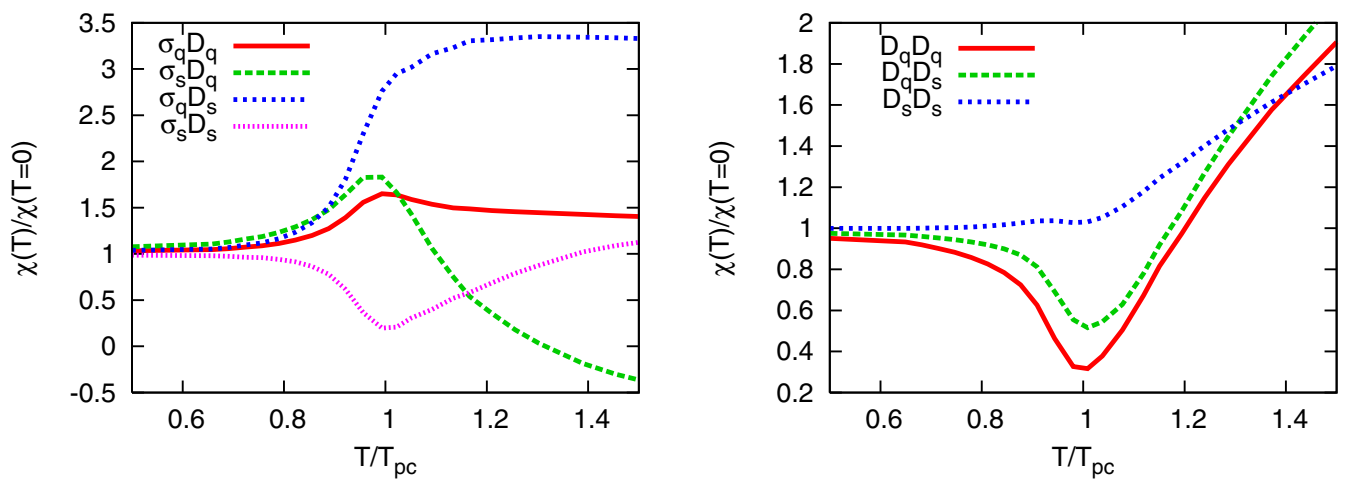

Figure 2. Flavor correlations between the light and heavy-light mesons (left) and among heavy-light mesons (right) [19].

Within the same theoretical framework, thermal fluctuations and correlations between the light and heavy-light mesons can also be studied. The extended susceptibility is given in the following matrix form [19]:

$$
\hat{\chi}_{\sigma \sigma}=\hat{\chi}_{\mathrm{ch}}+\hat{\chi}_{\mathrm{ch}} \hat{\mathcal{C}}_{\mathrm{HL}} \hat{\chi}_{D} \hat{C}_{\mathrm{HL}} \hat{\chi}_{\mathrm{ch}}, \quad \hat{\chi}_{\sigma D}=-\hat{\chi}_{\mathrm{ch}} \hat{\mathcal{C}}_{\mathrm{HL}} \hat{\chi}_{D}, \quad \hat{\chi}_{D D}=\hat{C}_{D}-\hat{C}_{\mathrm{HL}} \hat{\chi}_{\mathrm{ch}} \hat{C}_{\mathrm{HL}} \equiv \hat{\chi}_{D},
$$

where $\hat{\chi}_{\mathrm{ch}}$ is the chiral susceptibilities of non-strange and strange quark condensates which are responsible for the critical behaviors, and $\hat{C}_{\mathrm{HL}, D}$ represents the curvature of the effective potential.

Correlations between the light and heavy-light mesons, as well as those between the heavy-light mesons, are shown in Fig. 2. Due to the embedding of $\hat{\chi}_{\mathrm{ch}}$, various heavy-light flavor correlations are modified qualitatively in a narrow range of temperature where the chiral susceptibility exhibits a peak. The onset of the chiral crossover, in the heavy-light flavor correlations, is therefore independent from the light flavors. This indicates that the fluctuations carried by strange charmed mesons can also be used to identify the chiral crossover, which is dominated by the non-strange light quark dynamics.

It is apparent that the effective interactions between the light and charmed mesons play the central role in thermodynamics near the chiral crossover. The non-trivial thermal dependence was extracted with the help of the well-established quantities in lattice QCD. Physically, such an intrinsic thermal effect is carried by heavier hadronic states, or equivalently by the quarks and gluons with high frequencies. Those modes are integrated out and do not explicitly appear as local field operators in the Lagrangian. Toward the QCD phase transition, they become more activated and contribute to the ground state.

It is not straightforward to deal with many (or all the) hadrons. In holographic QCD approach, although infinite vector-mesons are naturally accommodated, a systematic technique to include $1 / N_{c}$ corrections in a medium is not established yet. In the standard effective theories in four dimensions, interactions with the higher-lying hadrons are not completely known. Integrating out the heavier modes at finite temperature and density is not an easy task either. Those effective interactions near the phase transition may be to some extent captured by use of more microscopic computations, e.g. lattice simulations, Dyson-Schwinger equations and functional approach. More elaborated and systematic prescription certainly requires a novel scheme, in particular at high density.

The chiral mass splittings are shown to be essentially insensitive to the light-quark flavors, in spite of a non-negligible explicit breaking of the chiral $S U(3)$ symmetry. This "blindness" of the charm 
quark to the light degrees of freedom is dictated by the heavy quark symmetry. In contrast, the kaon and its chiral partner masses become degenerate at a higher temperature than $T_{\mathrm{pc}}$, indicating a delay of the $S U(3)$ symmetry restoration. In the heavy-light sector, on the other hand, the strange charmed meson captures the onset of chiral symmetry restoration more strongly than the strange light meson does. A similar consequence is found in the correlations involving the heavy-light meson mean fields. Those fluctuations exhibit certain qualitative changes around $T_{\mathrm{pc}}$, and this feature is independent of the light flavors. Hence, the strange charmed mesons have a potential for measuring a remnant of $O(4)$.

It is a real challenge to explore the thermodynamics of heavy-light hadrons at high density. A central task is to introduce a reliable density dependence of the interaction parameters, which requires either a more microscopic prescription in the effective theory side, or more precise data for the chiral condensates from lattice simulations. There are some implications of a confined phase with unbroken chiral symmetry from the lattice studies where the low-lying Dirac modes, which generate a nonvanishing chiral condensate via the Banks-Casher relation, are removed. This new state of hadronic matter might appear in the QCD phase diagram at high baryon density, where chiral hadronic theories may become valid in a wider parameter range (See Ref. [20, 21] and references therein).

\section{Modified hadrons in hot and dense matter}

A recent study, comparing QCD thermodynamics obtained on the lattice with the hadron resonance gas (HRG) model [22], indicates that additional interaction strength, beyond that embodied by well established strange resonances [23], may be needed in the HRG model to remove disparities with the lattice results. In particular, the HRG results for the strangeness and mixed strangeness-baryon number susceptibilities $\left(\chi_{S S}\right.$ and $\left.\chi_{B S}\right)$ are clearly below those of the lattice, while the results for the thermodynamic pressure and the baryon number susceptibility are in good agreement.

This motivates the search for hitherto unknown strange hadrons, which could reduce or eliminate this discrepancy. In the PDG database, there are around twenty unconfirmed states with a mass below $2.0 \mathrm{GeV}$. Although these are not established resonances, the interactions in the corresponding scattering channels may yield important contribution to thermodynamic quantities.

More generally, a possible origin of the discrepancy is interaction strength in channels carrying net strangeness that so far have not been accounted for. Given the corresponding empirical scattering phase shifts, both confirmed and unconfirmed resonances as well as non-resonant interactions can be handled in a unified, model-independent way, using the S-matrix approach [24].

The strange scalar channel, with the unconfirmed $K_{0}^{*}(800)$ resonance, a.k.a. $\kappa$, is a prime candidate. Since the corresponding phase shifts for S-wave $K \pi$ scattering are fairly well determined, this channel is well suited for the S-matrix approach. In addition, the counterpart of $\kappa$ in the scalar-isoscalar channel, the $f_{0}(500)$, a.k.a. $\sigma$, though considered to be established [25], is unlike a typical resonance. Since the $\pi \pi \mathrm{S}$-wave phase shifts are known with reasonable accuracy, also this channel is a prime candidate for the S-matrix approach to thermodynamics. In Ref. [26], we focus on the strange scalar channel and its contribution to strangeness susceptibilities.

In Fig. 3-left, we show the strange susceptibility (normalized to $T^{2}$ ) computed in HRG using only established resonances (PDG, broken dashed line) and adding the unconfirmed $\kappa$ (PDG + kappa, full line). Indeed, within the treatment of the HRG model, we found that this single state alone accounts for the missing contribution in the strange susceptibility. However, owing to the large width of the $\kappa$ meson and the significant non-resonant background, the HRG model does not provide an accurate description of the interaction contributions. In fact, a consistent treatment of all $K \pi \mathrm{S}$-wave interactions within the S-matrix approach shows that a simplified (HRG) treatment of the interactions 

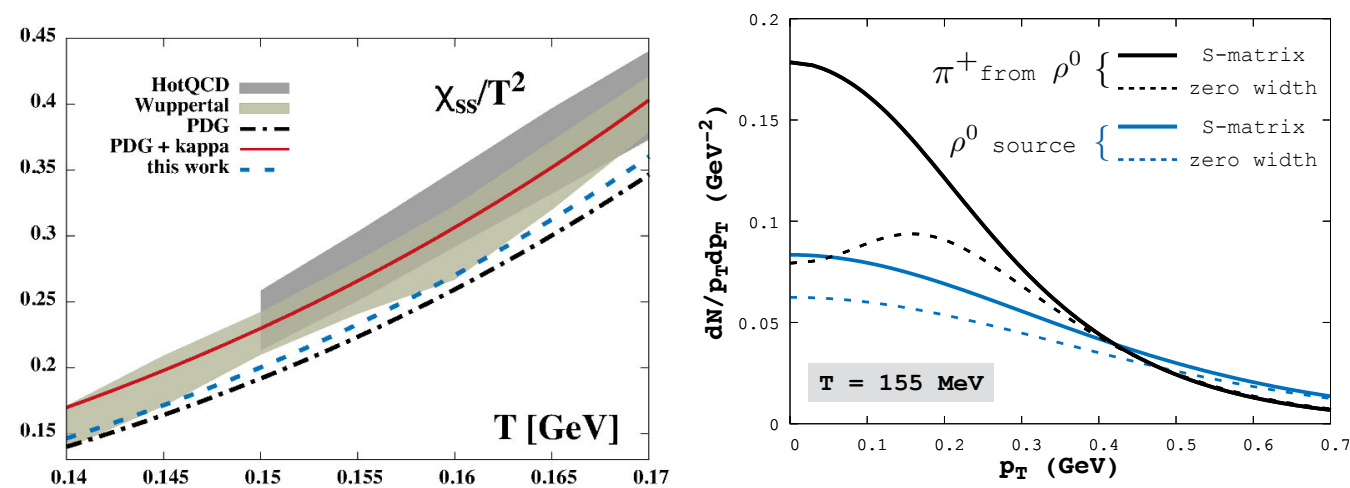

Figure 3. The strange susceptibility (left) [26] and the $p_{T}$ spectrum of $\rho^{0}$ in thermal equilibrium $(T=155 \mathrm{MeV})$ and the daughter $\pi^{+}$originating from $\rho^{0} \rightarrow \pi^{+} \pi^{-}$decay (right) [27].

in these channels, using a Breit-Wigner spectral function for each resonance and ignoring the nonresonant background, systematically overestimates the contribution to strangeness fluctuations.

In short, the $K \pi \mathrm{S}$-wave interactions provide only a part of the missing contribution to the strangeness susceptibility, indicated by recent lattice QCD results. Whether the remaining discrepancy can be resolved by a consistent treatment of other strangeness carrying channels will be explored in future studies.

The proper treatment of resonances which accounts for their production dynamics encoded in data for partial wave scattering amplitudes can substantially modify spectra of daughter particles originating in their two body decays. The influence of the finite width of $\rho$ meson on the pion momentum distribution results in an enhancement of the low- $p_{T}$ pions from the decays of $\rho$ mesons [27], as shown in Fig. 3-right. This improves the quantitative description of the pion spectra in heavy ion collisions obtained by the ALICE collaboration at the LHC energy [28].

The situation at high density is less clear [29]. Nevertheless, in the context of spontaneous chiral symmetry breaking, the quark-composition of the lowest scalar meson is naturally expected as a mixture of the quark-bilinear and tetraquark states. There is an interesting option of two different phases with broken chiral symmetry at finite density where a rather unorthodox pattern of spontaneous chiral symmetry breaking could be realized [30]. This pattern keeps the center of chiral group unbroken, i.e.

$$
S U\left(N_{f}\right)_{L} \times S U\left(N_{f}\right)_{R} \rightarrow S U\left(N_{f}\right)_{V} \times\left(Z_{N_{f}}\right)_{A},
$$

where a discrete symmetry $\left(Z_{N_{f}}\right)_{A}$ is the maximal axial subgroup of $S U\left(N_{f}\right)_{L} \times S U\left(N_{f}\right)_{R}$. The center $Z_{N_{f}}$ symmetry protects a theory from condensate of quark bilinears $\langle\bar{q} q\rangle$. Spontaneous symmetry breaking is driven by quartic condensates which are invariant under both $S U\left(N_{f}\right)_{V}$ and $Z_{N_{f}}$ transformation. In a system with the breaking pattern (3) the quartic condensate is the strict order parameter which separates different chirally-broken phases.

Assuming (3) at finite density, it is shown that an intermediate phase between chiral symmetry broken and its restored phases can be realized using a general Ginzburg-Landau free energy [31]. The pion decay constant is read from the Noether current as $F_{\pi}=\sqrt{\sigma_{0}^{2}+\frac{8}{3} \chi_{0}^{2}}$ with $\chi_{0}$ and $\sigma_{0}$ being the expectation values of 4-quark and 2-quark scalar fields, determined from the gap equations. The 

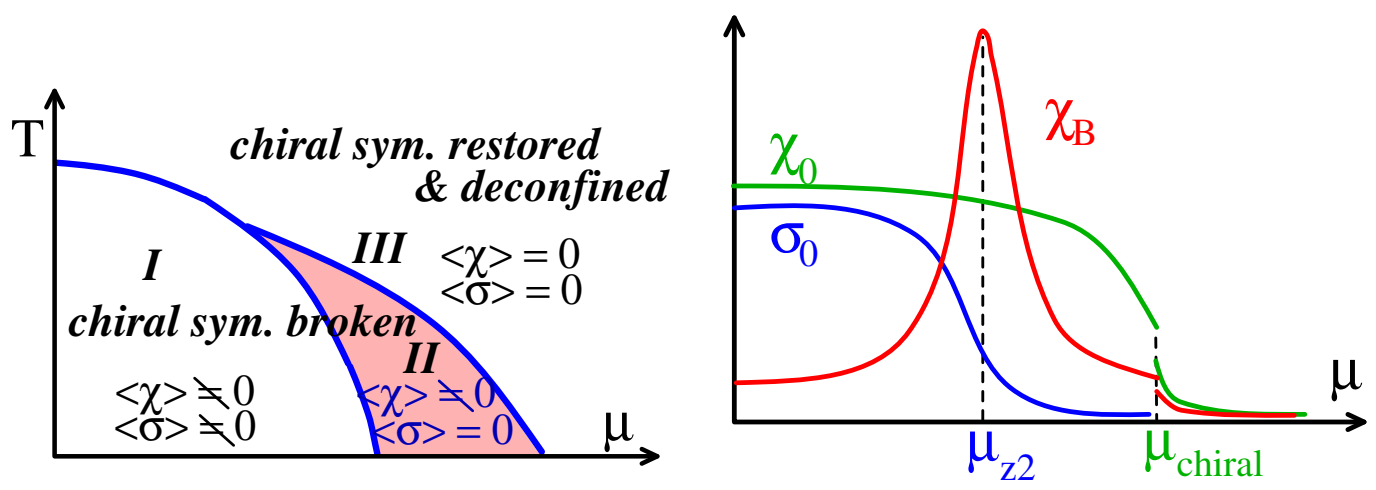

Figure 4. Schematic phase diagram mapped onto $(T, \mu)$ plane (left) and a sketch of the baryon number susceptibility as a function of chemical potential assuming a first-order chiral transition (right) [31].

effective potential deduced in the mean field approximation describes three distinct phases characterized by the two order parameters: Phase I represents the system where both chiral symmetry and its center are spontaneously broken due to non-vanishing expectation values $\chi_{0}$ and $\sigma_{0}$. The center symmetry is restored when $\sigma_{0}$ becomes zero. However, chiral symmetry remains broken as long as $\chi_{0}$ is non-vanishing, where the pure 4-quark state is the massless Nambu-Goldstone boson (phase II). The chiral symmetry restoration takes place under $\chi_{0} \rightarrow 0$ which corresponds to phase III. With an explicit breaking of chiral symmetry one would draw a phase diagram mapped onto $(T, \mu)$ plane as summarized in Fig. 4-left.

A characteristic feature with unbroken center symmetry is found in the baryon number susceptibility. The $Z_{2}$ invariance prohibits a Yukawa coupling of $\chi$ to baryons. Consequently, the baryon number susceptibility exhibits a maximum when across the boundary of $Z_{2}$ restoration (see Fig. 4-right). The mass spectra of the low-lying mesons and baryons will be modified in phase II. A systematic classification based on the relevant symmetry done for $N_{f}=2$ and $N_{f}=3$ [31] can be extrapolated to the realistic $N_{f}=2+1$ case: one would naturally expect for the non-strange scalar and pseudo-scalar mesons and the nucleon parity doublers

$$
m_{S} \neq m_{P}, \quad m_{N_{+}} \simeq m_{N_{-}}
$$

and for the strange mesons and baryons

$$
m_{S_{s}} \simeq m_{P_{s}}, \quad m_{B_{+}} \sim m_{B_{-}} .
$$

Therefore, the early onset of (partial) chiral symmetry restoration would be seen in the strange-hadron. We note that the vector and axial-vector mesons remain non-degenerate both in the non-strange and strange sector.

\section{Conclusions}

Various fluctuations of conserved charges as well as the Polyakov-loop susceptibilities in lattice QCD $[32,33]$ consistently indicate that deconfinement takes place in the chiral crossover region at 
vanishing chemical potential. Although the kurtosis of net quark number fluctuations is reasonably quantified in a class of chiral models with the Polyakov loop [34], modifications in the Polyakov loop fluctuations by the dynamical quarks cannot be explained in the same framework. Since those models do not posses the dynamical mechanism for quark confinement, the properties of gluon-oriented quantities are supposed to be less captured. An effective theory that can better handle the confinement nature of non-abelian gauge theories is indispensable to reveal the Polyakov loop fluctuations in the presence of light quarks. Also, it is vital to bridge the gap between the Dirac zero eigenmodes, responsible for dynamical chiral symmetry breaking, and the magnetic monopoles.

The importance of higher-lying hadrons has been found in low-energy constants and spectral functions near the QCD phase transition. On a practical level, it is not yet established to fully accommodate them to effective theories. Several attempts constrained by the relevant global symmetries lead to certain non-trivial medium effects. More elaborated and systematic prescription certainly requires a novel scheme. Functional approaches in terms of quarks and gluons may provide some benefits in this context.

Heavy-light hadrons, such as charmed mesons, are also good probes for the quark-gluon dynamics. In dilute nuclear matter, in-medium modifications of the color-electric and color-magnetic gluons are extracted from the D and B meson dynamics [35]. In increasing density/temperature, those heavylight mesons will change their chiral properties, as expected from the chiral doubling scenario [3638]. The mass gap between the chiral partners is around $350 \mathrm{MeV}$, and this is much bigger than a mass difference between the charged D mesons in dense matter, $50 \mathrm{MeV}$. Further theoretical investigations will supply more reliable understanding of heavy-flavor transport properties.

\section{Acknowledgments}

The work has been partly supported by the Polish Science Foundation (NCN) under Maestro grant DEC-2013/10/A/ST2/00106.

\section{References}

[1] S. Jeon and V. Koch, In *Hwa, R.C. (ed.) et al.: Quark gluon plasma* 430-490.

[2] P. Braun-Munzinger and J. Stachel, Phys. Lett. B 490, 196 (2000).

[3] M. Gazdzicki, Phys. Rev. C 60, 054903 (1999).

[4] M. Gazdzicki and M. Gorenstein, Phys. Rev. Lett. 83, 4009 (1999).

[5] A. Adare et al. [PHENIX Collaboration], Phys. Rev. C 84, 044905 (2011).

[6] L. Adamczyk et al. [STAR Collaboration], arXiv:1404.6185 [nucl-ex].

[7] B. Abelev et al. [ALICE Collaboration], JHEP 1209, 112 (2012).

[8] B. Abelev et al. [ALICE Collaboration], Phys. Rev. Lett. 111, 102301 (2013).

[9] A. Bazavov, H.-T. Ding, P. Hegde, O. Kaczmarek, F. Karsch, E. Laermann, Y. Maezawa and S. Mukherjee et al., Phys. Lett. B 737, 210 (2014).

[10] N. Isgur and M. B. Wise, Phys. Lett. B 232, 113 (1989); Phys. Lett. B 237, 527 (1990).

[11] H. Georgi, Phys. Lett. B 240, 447 (1990).

[12] M. B. Wise, Phys. Rev. D 45, 2188 (1992).

[13] G. Burdman and J. F. Donoghue, Phys. Lett. B 280, 287 (1992).

[14] T. M. Yan, H. Y. Cheng, C. Y. Cheung, G. L. Lin, Y. C. Lin and H. L. Yu, Phys. Rev. D 46, 1148 (1992) [Erratum-ibid. D 55, 5851 (1997)].

[15] M. A. Nowak, M. Rho and I. Zahed, Phys. Rev. D 48, 4370 (1993). 
[16] W. A. Bardeen and C. T. Hill, Phys. Rev. D 49, 409 (1994).

[17] C. Sasaki, Phys. Rev. D 90, no. 11, 114007 (2014).

[18] D. Roder, J. Ruppert and D. H. Rischke, Phys. Rev. D 68, 016003 (2003).

[19] C. Sasaki and K. Redlich, Phys. Rev. D 91, no. 7, 074021 (2015)

[20] C. Sasaki, Nucl. Phys. A 931, 238 (2014).

[21] T. M. Doi, K. Redlich, C. Sasaki and H. Suganuma, Phys. Rev. D 92, no. 9, 094004 (2015).

[22] A. Bazavov, H.-T. Ding, P. Hegde, O. Kaczmarek, F. Karsch, E. Laermann, Y. Maezawa and

S. Mukherjee et al., Phys. Rev. Lett. 113, no. 7, 072001 (2014).

[23] K. A. Olive et al. [Particle Data Group Collaboration], Chin. Phys. C 38, 090001 (2014).

[24] R. Dashen, S. K. Ma and H. J. Bernstein, Phys. Rev. 187, 345 (1969).

[25] J. R. Pelaez, PoS ConfinementX , 019 (2012).

[26] B. Friman, P. M. Lo, M. Marczenko, K. Redlich and C. Sasaki, Phys. Rev. D 92, no. 7, 074003 (2015).

[27] P. Huovinen, P. M. Lo, M. Marczenko, K. Morita, K. Redlich and C. Sasaki, arXiv:1608.06817 [hep-ph].

[28] B. Abelev et al. [ALICE Collaboration], Phys. Rev. C 88 (2013) 044910.

[29] K. Fukushima and C. Sasaki, Prog. Part. Nucl. Phys. 72, 99 (2013).

[30] I. I. Kogan, A. Kovner and M. A. Shifman, Phys. Rev. D 59, 016001 (1999).

[31] M. Harada, C. Sasaki and S. Takemoto, Phys. Rev. D 81, 016009 (2010).

[32] P. M. Lo, B. Friman, O. Kaczmarek, K. Redlich and C. Sasaki, Phys. Rev. D 88, 014506 (2013).

[33] P. M. Lo, B. Friman, O. Kaczmarek, K. Redlich and C. Sasaki, Phys. Rev. D 88, 074502 (2013).

[34] V. Skokov, B. Friman and K. Redlich, Phys. Rev. C 83, 054904 (2011).

[35] S. Yasui and K. Sudoh, Phys. Rev. C 89, 015201 (2014).

[36] M. A. Nowak, M. Rho and I. Zahed, Phys. Rev. D 48, 4370 (1993).

[37] W. A. Bardeen and C. T. Hill, Phys. Rev. D 49, 409 (1994).

[38] M. Harada, M. Rho and C. Sasaki, Phys. Rev. D 70, 074002 (2004). 